\title{
NEUROTOXICIDAD ALCOHÓLICA
}

\section{ANTONIO SCHLESINGER PIEDRAHITA ${ }^{1}$, BEATRIZ PESCADOR VARGAS ${ }^{2}$, LAURA ALEJANDRA ROA CULMA ${ }^{3 *}$}

\author{
${ }^{1}$ Médico, Neurólogo. Coordinador Servicio de Neurología. Clínica Shaio. \\ Bogotá - Colombia \\ ${ }^{2}$ Magister en Biología, Docente Asociada Facultad de Medicina \\ Universidad Militar Nueva Granada- Bogotá - Colombia \\ ${ }^{3}$ Bióloga Egresada de la Universidad del Tolima - Colombia \\ *Correspondencia: lauraroac14@gmail.co
}

Recibido: 3 Junio 2016 Aceptado: 13 Febrero 2017

\section{RESUMEN}

El alcohol es un tóxico que, actúa como depresor del sistema nervioso central (SNC). Sus efectos son consecuencia directa de su acción sobre las membranas celulares y sobre los neurotransmisores. Las neuronas son particularmente sensibles al efecto neurotóxico del Etanol (EtOH) y su exposición afecta la morfología neuronal, ya que en estudios recientes se ha observado una disminución en la longitud del árbol dendrítico. El EtOH también produce cambios en la expresión de las proteínas sinápticas, aumento del lumen del retículo endoplasmático, así como también, desorganización del aparato de Golgi y de las mitocondrias.

Objetivos: Describir los efectos del Etanol a nivel celular y molecular en el Sistema Nervioso Central y su repercusión en la salud humana.

Métodos: Se realizó una búsqueda de literatura en las siguientes bases de datos disponibles en web: PubMed, ScienceDirect, Jama Journal, Scielo y portales de salud, utilizando las palabras claves: "etanol y farmacología", "alcohol y toxicología", "neurotoxicidad y etanol".

Resultados: De las bases consultadas se obtuvo un total de 120 referencias de las cuales se aceptaron solo 55 que resaltan los aspectos más relevantes de la fisiopatología de la intoxicación con etanol y ciertos aspectos a nivel molecular relacionados con los efectos sobre el SNC.

Conclusiones: Los efectos del EtOH sobre el sistema nervioso pueden ser consecuencia de un consumo agudo y excesivo o secundario a un consumo crónico, en relación a un estado de adicción y dependencia al mismo. La toxicidad sobre el sistema nervioso es consecuencia directa de la agresión del etanol o por diferentes trastornos metabólicos ligados a la toxicidad del mismo sobre otros órganos. La neurotoxicidad del alcohol se debe a la activación del sistema inmunitario innato en el cerebro, que promueve compuestos inflamatorios que serían los responsables del daño de la morfología neuronal, además de la alteración neuroanatómica de la corteza.

Palabras Clave: Alcoholismo; Neurotoxicidad; Sinapsis; Muerte Celular; Apoptosis. 


\title{
ALCOHOLIC NEUROTOXICITY
}

\begin{abstract}
Alcohol is a toxic that acts as a central nervous system (CNS) depressant. Its effects are a direct consequence of its action on cell membranes and on neurotransmitters. Neurons are particularly sensitive to the neurotoxic effect of Ethanol $(\mathrm{EtOH})$ and their exposure affects neuronal morphology, since a reduction in the length of the dendritic tree has recently been observed. EtOH also causes changes in the expression of synaptic proteins, increased endoplasmic reticulum light, as well as disorganization of the Golgi apparatus and mitochondria.

Objectives: To describe the effects of Ethanol at the cellular and molecular level in the Central Nervous System and its repercussion on human health.

Methods: A literature search was conducted in the following databases available on the web: PubMed, ScienceDirect, Jama Journal, Scielo and health portals, using the keywords: "ethanol and pharmacology", "alcohol and toxicology", " Neurotoxicity and ethanol. "

Results: From the databases consulted, a total of 120 references were obtained, of which 55 are accepted, highlighting the most relevant pathophysiology of ethanol intoxication and the minimum number of molecular levels related to the effects on the CNS.

Conclusions: The effects of EtOH on the nervous system can be a consequence of an acute and excessive consumption or secondary to a chronic consumption, in relation to a state of addiction and dependence to it. The toxicity on the nervous system is the direct consequence of the aggression of ethanol and the different metabolic disorders linked to the toxicity of the same on other organs. The neurotoxicity of alcohol is due to the activation of the immune system in the brain, which promotes inflammatory cascade that cause those responsible for neuron damage, in addition to neuroanalytic alteration of the cortex.
\end{abstract}

Keywords: Alcoholism; Neurotoxicity; Synapse; Cell death; Apoptosis. 


\section{NEUROTOXICIDADE ALCOÓLATRA}

\section{RESUMO}

O álcool é um tóxico que atua como um depressor do sistema nervoso central (SNC). Seus efeitos são conseqüência direta de sua ação nas membranas celulares e nos neurotransmissores. Os neurônios são particularmente sensiveis ao efeito neurotóxico do Etanol (EtOH) e sua exposição afeta a morfologia neuronal, uma vez que uma redução no comprimento da árvore dendrítica foi recentemente observada. EtOH também provoca alterações na expressão de proteínas sinápticas, aumento da luz do retículo endoplasmático, bem como desorganização do aparelho de Golgi $e$ das mitocôndrias.

Objetivos: Descrever os efeitos do Etanol no nível celular e molecular no Sistema Nervoso Central e sua repercussão na saúde humana.

Métodos: Uma investiga çao bibliográfica foi realizada nos seguintes bancos de dados disponiveis na web: PubMed, ScienceDirect, Jama Journal, Scielo E portais de saúde, usando as palavras-chave:"ethanol and pharmacology", "Álcool e toxicologia", "Neurotoxicidade e etanol".

Resultados: partir dos bancos de dados consultados, foram obtidas 120 referências, das quais 55 são aceitas, destacando a fisiopatologia mais relevante da intoxicação por etanol e o número mínimo de níveis moleculares relacionados aos efeitos no SNC.

Conclusões: Os efeitos do EtOH no sistema nervoso podem ser uma conseqüência de um consumo agudo e excessivo ou secundário a um consumo crônico, em relação a um estado de dependência e dependência. A toxicidade no sistema nervoso é a conseqüência direta da agressão do etanol e dos diferentes distúrbios metabólicos ligados à toxicidade do mesmo em outros órgãos. A neurotoxicidade do álcool deve-se à ativação do sistema imunológico no cérebro, que promove a cascata inflamatória que causa os responsáveis pelo dano neuronal, além da alteração neuroanalítica do córtex.

Palavras-chave: Tumor; pâncreas; mulheres; tomografia. 


\section{Introducción}

El alcohol etílico o etanol ( $\left.\mathrm{EtOH} ; \mathrm{C}_{2} \mathrm{H}_{6} \mathrm{O}\right)$ es un líquido incoloro, translucido, de olor característico, excelente solvente, menos denso que el agua $(\delta=0,789 \mathrm{~g} / \mathrm{ml})$, con un peso molecular (PM) de 46,0634 daltons (1). Es una sustancia psicoactiva que afecta al cerebro, la conducta y la cognición, actuando como depresor del sistema nervioso central; su consumo crónico y excesivo se asocia a numerosas enfermedades inflamatorias y degenerativas que pueden acabar con la vida del consumidor debido a efectos sobre el sistema cardiovascular ocasionando miocardiopatía alcohólica; el páncreas, causando pancreatitis aguda y crónica; nervios periféricos manifestándose como polineuropatía alcohólica; sistema músculo-esquelético ocasionando osteoporosis y miopatía alcohólica; sobre el sistema nervioso central causando atrofia cerebral y cerebelosa, encefalopatías y sobre el feto bajo la patología del síndrome alcohólico fetal; además de hipoglucemias, hepatitis aguda, rabdomiolisis y enfermedades psiquiátricas como ansiedad y depresión $(2,3)$.

La Organización Mundial de la Salud (OMS) define el alcoholismo (alcohol-dependencia), como el consumo crónico y continuado o periódico de alcohol que se caracteriza por un deterioro del control sobre la bebida, episodios frecuentes de intoxicación, obsesión por el mismo y consumo a pesar de sus consecuencias adversas (4-6).

Esta sustancia puede ser liposoluble e hidrosoluble, de esta manera le confiere la capacidad de poder difundir fácilmente a través de la membrana celular estableciendo un enlace apolar en las cadenas de hidrocarburos, afectando el potencial de acción de las fibras nerviosas ya que interfiere con la permeabilidad de iones como sodio y potasio; de igual forma le permite atravesar la barrera hematoencefálica (BHE), fetoplacentaria y de distribuirse a través de todos los fluidos y tejidos corporales (7).

A su vez el EtOH interfiere con los procesos de transporte activo a nivel celular, ya que disminuye la actividad de la ATP-asa a nivel de las membranas, ocasionando el efecto primario del alcohol sobre el tejido nervioso que es la disminución de la excitabilidad (8).

El consumo de $\mathrm{EtOH}$, se relaciona con más de 60 condiciones de salud, desde las que son resultado de un consumo excesivo durante el embarazo y que afectan al feto, hasta lesiones intencionales, enfermedades hepáticas y condiciones neurológicas, ya que el tejido ner- vioso es particularmente sensible al efecto del etanol, afectando la anatomía neuronal y causando cambios en la expresión de proteínas, llevando finalmente a la muerte celular y necrosis (9). De igual forma, se relaciona con el desarrollo de eventos epidémicos - cardiovasculares, cáncer y otros que se están incrementando- con enfermedades sociales y mentales, con la violencia intrafamiliar, la morbilidad y mortalidad por accidentes de tráfico, el suicidio, el ausentismo laboral y escolar, los problemas interpersonales y las conductas sexuales peligrosas (10). Por lo anterior, el objetivo del presente artículo es describir los efectos del Etanol a nivel celular y molecular en el Sistema Nervioso Central (SNC) y su repercusión en la salud humana.

\section{Método}

Se realizó una revisión en la literatura de los principales aspectos del metabolismo del alcohol y sus productos, que intervienen como factores de riesgo para el desarrollo de múltiples alteraciones que llevan a degeneración neuronal. La búsqueda de material bibliográfico se llevó a cabo a través de las bases de datos disponibles en la web tales como PubMed, ScienceDirect, Dialnet, Jama Journal, Scielo y portales de salud como la Organización Mundial de la Salud (OMS), utilizando las palabras claves: "etanol y farmacología", "alcohol y toxicología", "neurotoxicidad y etanol". Como criterios de inclusión se planteó la elección de los artículos que informaran sobre aspectos bioquímicos, farmacológicos y estudios clínicos y experimentales.

\section{Resultados}

Se obtuvieron 120 referencias de las cuales se filtraron para obtener únicamente aquellas que evidenciaran relevancia en los aspectos a tratar y profundizar en el presente artículo, quedando un total de 55 artículos en los cuales se resaltan los aspectos más relevantes de la neuroanatomía de la intoxicación con etanol y ciertos aspectos a nivel molecular relacionados con los efectos sobre el SNC.

Se obtuvieron 120 referencias de las cuales se filtraron para obtener únicamente aquellas que evidenciaran relevancia en los aspectos a tratar y profundizar en el presente artículo, quedando un total de 55 artículos en los cuales se resaltan los aspectos más relevantes de la neuroanatomía de la intoxicación con etanol y ciertos aspectos a nivel molecular relacionados con los efectos sobre el SNC. 


\section{Farmacocinética}

El etanol puede administrarse de diversas formas y absorberse por múltiples vías; no es un producto normal del metabolismo, aunque se producen cantidades mínimas en el intestino por fermentación de la flora bacteriana (11). Sin embargo, su principal vía de ingreso suele ser oral, mediante el cual es absorbido a lo largo del tracto gastrointestinal fundamentalmente la primera porción del intestino delgado, aproximadamente en un $70 \%$; el estómago en un $20 \%$ y el colon en un $10 \%$ (7).

La velocidad de absorción depende de diferentes factores, como la presencia o ausencia de alimentos, el tiempo de vaciamiento gástrico (la presencia de azúcar y ácido carbónico favorecen la absorción, mientras que una comida rica en contenidos grasos la retardan); la cantidad de alcohol ingerida (bebidas de alto contenido alcohólico tienen una absorción más lenta, debido a que aumenta el tono pilórico), entre otros; llegando a alcanzar su máximo nivel sanguíneo entre 60 y 90 minutos después su ingestión (12).

Por vía dérmica e inhalatoria también se puede absorber, aunque ésta es limitada; la vía endovenosa se utiliza de manera terapéutica en el tratamiento de la intoxicación por alcohol metílico o por etilenglicol $(13,14)$.

El etanol posee un coeficiente de partición de 0.5, aunque en el organismo se distribuye con mayor facilidad en los medios acuosos; al ser una molécula de carga débil pasa con rapidez a través de la membrana celular y se equilibra en la sangre; presenta una relación entre el líquido intravascular y extravascular de 1:25 (15). Dicha sustancia se concentra principalmente en tejidos como el cerebro, sangre, ojo y líquido cefalorraquídeo; presentando un equilibrio en aquellos tejidos que son altamente irrigados: cerebro, hígado, riñón y pulmones (11).

Inmediatamente llega el $\mathrm{EtOH}$ a la sangre se inicia su eliminación a través del metabolismo hepático y en menor proporción en otros lugares como la mucosa intestinal o también mediante la excreción intercambiada; sólo un $10 \%$ del etanol absorbido se excreta por ésta última vía, a través del aliento, la saliva, las heces, orina, sudor o la leche (16).

El EtOH desaparece del plasma, con una cinética de orden cero, entre las 10-12 horas posteriores a la finalización de la ingesta. El 90-98\% del EtOH ingerido se oxida completamente a una velocidad de 100-150 $\mathrm{mg} / \mathrm{kg} / \mathrm{h}$. Los alcohólicos crónicos, en tanto tengan una función hepática conservada, pueden presentar un aumento de la velocidad de oxidación; por otro lado, la alcoholemia de una persona que tiene daño hepático puede permanecer elevada más allá de las 24 horas de finalizada la última ingesta (17).

En el hígado se produce la metabolización del 85-90\% del EtOH ingerido; un $70 \%$ se metaboliza por medio de la acción secuencial de las enzimas alcohol deshidrogenasa $(\mathrm{ADH})$ y aldehído deshidrogenasa (ALDH), el llamado Sistema alcohol deshidrogenasa (ADH), en su mayoría lo oxida primero a acetaldehído (etanal) por acción de la enzima alcohol deshidrogenasa citosólica dependiente de $\mathrm{NAD}^{+}$; con una nueva oxidación, dicho compuesto es convertido en acetato por la enzima aldehído deshidrogenasa mitocondrial. El acetato es activado a acetil CoA por medio de la enzima acetato-CoA-ligasa con consumo de ATP; a partir de alli se degrada predominantemente en el ciclo de Krebs a $\mathrm{CO}_{2}$ y $\mathrm{H}_{2} \mathrm{O}(18,19)$.

Han sido identificadas 5 clases de isoenzimas de la $\mathrm{ADH}$ hepática $(\alpha, \beta, \gamma, \pi, \mathrm{X})$, con distinta afinidad por el EtOH y codificadas en 7 locus genéticos diferentes; se encuentran agrupadas en tres clases: ADH clase I (subunidades $\alpha, \beta, \gamma$ ), ADH clase II (subunidad $\pi$ ) y ADH clase III (subunidad X) (20). La velocidad de la degradación del $\mathrm{EtOH}$ en el hígado está limitada por la actividad de la enzima alcohol deshidrogenasa que a su vez depende de la cantidad disponible de NAD+ como factor limitante. A concentraciones bajas de $\mathrm{EtOH}$ se alcanza una velocidad de degradación máxima, es por esto que a velocidad constante el nivel de $\mathrm{EtOH}$ disminuye (cinética de orden 0). En promedio, un individuo adulto puede metabolizar entre 7 y 10 gramos de alcohol por hora (19). Sin embargo, esto cambia entre individuos y depende de los polimorfismos de la enzima alcohol deshidrogenasa (8). Se postula que el polimorfismo en dichas enzimas podría ser responsable de las diferencias metabólicas que se observan entre los sexos, diferentes individuos y grupos étnicos. También serían responsables de las distintas susceptibilidades o resistencias a desarrollar dependencia y/o tolerancia al consumo de $\mathrm{EtOH}$ (19-21).

Aproximadamente $10-30 \%$ del EtOH se oxida por medio del denominado sistema de oxidación del EtOH u oxidasas microsomales (Sistema microsomal oxidativo del etanol MEOS), involucra la fracción CYP2E1 del citocromo $\mathrm{P}_{450}$ y requiere $\mathrm{NADPH}$, en lugar de $\mathrm{NAD}^{+}$, se encuentra en el retículo endoplasmático del hepatocito $y$ es el principal mecanismo de adaptación en el alcoholismo crónico, cuando se encuentra saturada la capacidad 
de la anterior vía $(\mathrm{ADH})(18,20)$. La hipertrofia de este sistema provoca un exceso de radicales libres (anión superóxido $\mathrm{O}^{2}$, peróxido de hidrógeno $\mathrm{H}_{2} \mathrm{O}_{2}$, radical hidroxilo $\mathrm{OH}$ ) y el consiguiente estrés oxidativo con daño del hepatocito. Debido a que los fármacos son metabolizados en los microsomas, el EtOH interferirá en el metabolismo de los mismos, lo que explicaría las interacciones alcohol-fármacos (21).

Un tercer sistema de metabolización es el de la catalasa, enzima presente en los peroxisomas, que oxida al EtOH utilizando peróxido de hidrógeno $\left(\mathrm{H}_{2} \mathrm{O}_{2}\right)$. La tasa fisiológica de producción de $\mathrm{H}_{2} \mathrm{O}_{2}$ es pequeña, lo que indica que la catalasa sólo representaría alrededor del $2 \%$ de la tasa in vivo de oxidación del etanol (20). Este sistema parecería ejercer su acción sólo ante concentraciones elevadas de la droga y ser de escasa importancia en el metabolismo hepático, no así en el cerebro $(15,22)$ (Figura 1).

El acetaldehído formado como metabolito intermedio en todos los sistemas del metabolismo del $\mathrm{EtOH}$, es una molécula muy reactiva, responsable de gran parte de los efectos tóxicos atribuidos al propio EtOH. El 2-10\% del EtOH que escapa a la oxidación se elimina, sin modificar, por vía renal y pulmonar (principalmente); y en virtud de su capacidad para atravesar membranas, se lo puede encontrar en todas las secreciones corporales (8).

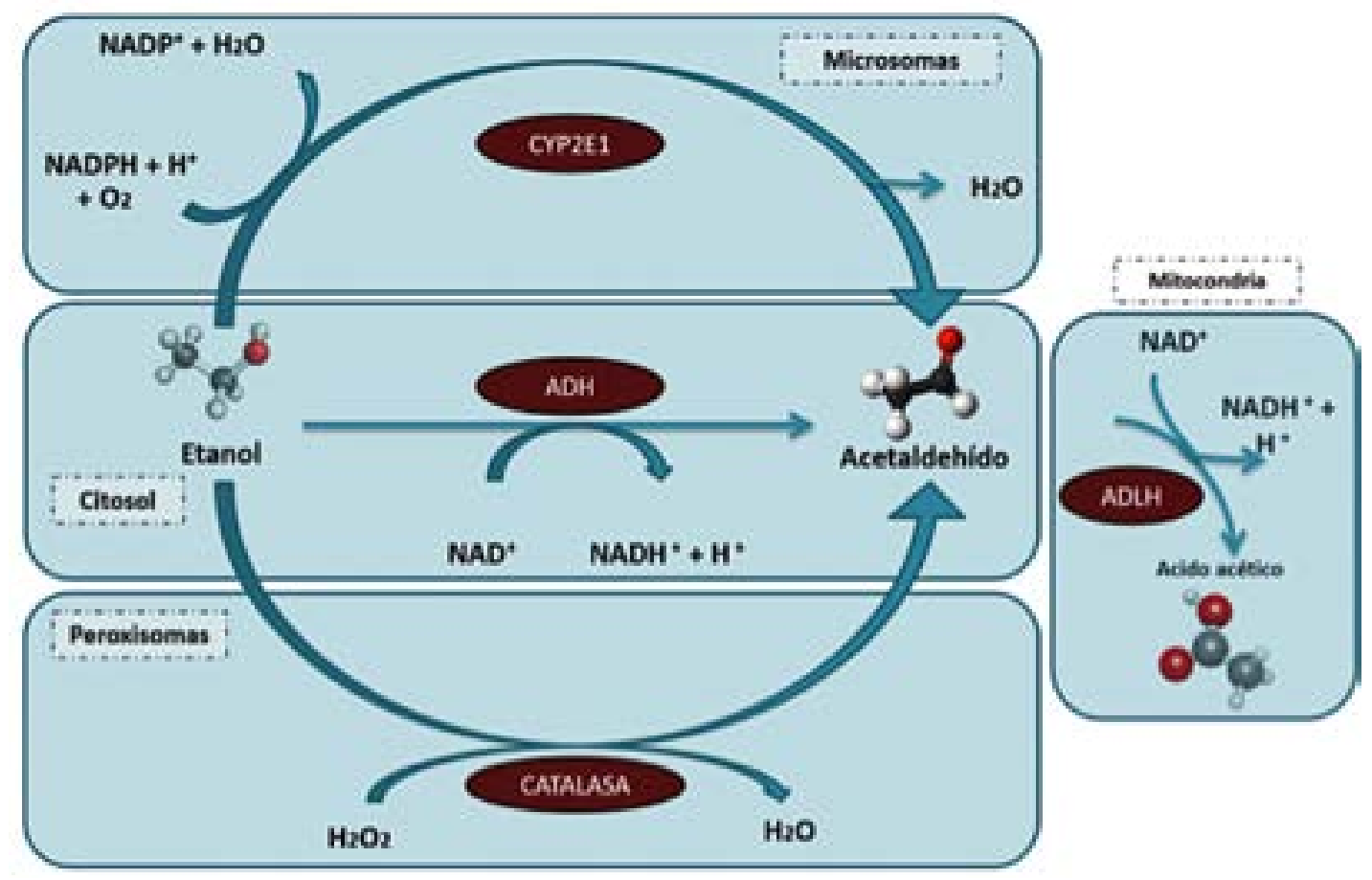

Figura 1. Metabolismo hepático del etanol. ADH: Alcohol deshidrogenasa.

ADLH: Aldehído deshidrogenasa. CYP2E1: citocromo P450-2E1.

Modificado de: www.asturnatura.com/articulos/nutricion/energia-nutrientes-componentes-dieta/alcohol.php

\section{Farmacodinamia}

$\mathrm{El} \mathrm{EtOH} \mathrm{actúa} \mathrm{de} \mathrm{manera} \mathrm{inespecífica} \mathrm{sobre} \mathrm{la} \mathrm{membra-}$ na neuronal alterando su permeabilidad, debido al efecto sobre las propiedades de fluidez y desorganización de sus componentes lipídicos, reducción de los fosfolípidos poliinsaturados e incremento del colesterol, lo cual se relaciona con el efecto depresivo central que el EtOH ofrece (1).
En el cerebro adulto, las sinapsis GABAérgicas son las principales fuentes de inhibición y las sinapsis que usan glutamato, las de excitación. Ambas operan a través de canales receptores ionotrópicos que son permeables a aniones y cationes respectivamente (23).

El ácido y-aminobutírico (GABA) es el principal neurotransmisor inhibidor SNC; desempeña el papel princi- 
pal en la reducción de excitabilidad neuronal a lo largo del sistema nervioso. En humanos, el GABA es directamente responsable de la regulación del tono muscular, afectando directamente los sistemas transmisores, especialmente sobre la sinapsis del sistema GABAérgico, como último eslabón responsable de la adquisición de la tolerancia al alcohol $(24,25)$. El EtOH posee la capacidad de aumentar la inhibición sináptica del GABA actuando sobre el receptor $\mathrm{GABA}_{\mathrm{A}}$, ampliamente distribuido en el tejido nervioso central; además a nivel de los receptores de aminoácidos excitatorios como el Glutamato, actúa sobre los receptores NMDA (N-metil-D-aspartato) y promueve la inhibición de corrientes de iones activadas por dicho neurotransmisor produciendo una up regulation, es decir, un aumento en el número de receptores que persiste, aunque el organismo esté libre de alcohol, lo cual puede ser el origen de las alucinaciones y convulsiones (26-29).

\section{Efectos del alcohol EtOH sobre el SNC}

El EtOH es un depresor del SNC, sin embargo, al comienzo resulta ser un estimulante debido a la depresión de mecanismos inhibidores centrales; en la intoxicación alcohólica aguda se produce un grado variable de estimulación del sistema nervioso (regocijo, excitación, desinhibición, locuacidad, agresividad, irritabilidad, descoordinación), pero en intoxicación intensa, puede seguirse de una fase inhibitoria que conduce finalmente a coma y en casos severos a muerte por depresión cardio-respiratoria $(30,31)$.

Esta sustancia es capaz de atravesar la BHE en un 90\%, por esta razón se le atribuye un efecto directo sobre el cerebro, interfiriendo incluso en los potenciales de acción nerviosa, desencadenando efectos sobre la conducta a causa de alteraciones en la corteza cerebral, sobre la electrofisiología del cerebro (potenciales evocados visuales y auditivos), el metabolismo cerebral y la circulación del mismo $(5,32)$.

Cuando las concentraciones de alcohol son altas, se incrementa la depresión funcional de la célula nerviosa por inhibición de la respiración tisular, reduciendo el metabolismo oxidativo y con ello los fosfatos ricos en energía (9); esto conlleva a alteraciones en los procesos mentales que dependen de la capacitación y la experiencia, que posteriormente repercute en la disminución de la memoria y la concentración. Estas alteraciones en la memoria se deben al fallo para adquirir nueva información y no a la recuperación de la información previamente adquirida; es probable que implique regiones cerebrales necesarias para el uso de la memoria de trabajo, tales como el hipocampo y la corteza prefrontal (33).

En el consumo crónico están implicados varios factores: cambios químicos en el cerebro que conllevan a la adaptación por el uso prolongado de alcohol; factores genéticos que codifican neurotransmisores y sus receptores; efectos psicológicos y fisiológicos, deprimiendo muchos centros neuronales (26-29).

En cuanto al metabolismo, la ADH clase I, que es la principal oxidante del etanol a concentraciones bajas y moderadas en el hígado, tiene una acción limitada en el SNC (34). Principalmente, en cerebro de humanos la isoforma más abundante de esta enzima es la clase III, sin embargo, presenta baja afinidad por el etanol y difícilmente es activada por éste, ni siquiera bajo severas intoxicaciones etílicas se alcanzan las concentraciones necesarias para que su contribución sea relevante $(35,36)$.

Por otro lado, se ha descrito la presencia de citocromos pertenecientes al complejo enzimático MEOS: CP450 (CYP2E1) a nivel cerebral, concentrándose principalmente en neuronas del córtex cerebral, células de Purkinje y granulares del cerebelo, el giro dentado y el hipocampo; a pesar de la pequeña cantidad de etanol oxidado en el cerebro, la producción de acetaldehído, secundario a estas reacciones, conlleva a importantes cambios en la fisiología y actividad de determinados grupos neuronales $(37,38)$.

\section{Efectos de la exposición al etanol sobre la morfo- logía neuronal}

Estudios realizados en pacientes alcohólicos han reportado daño selectivo de un 15 a $23 \%$ de poblaciones neuronales específicas, en los cuales se documenta la pérdida y contracción de neuronas piramidales del hipocampo, células de Purkinje del cerebelo y en particular de la sustancia blanca (36-38). Esto conlleva a un aumento en el número de células de la glía de la corteza frontal superior, como causa de la degeneración progresiva y muerte neuronal cortical y la subsiguiente proliferación de astrocitos como fenómeno de reparación (39).

Estudios neuro-radiológicos han demostrado que la contracción del cerebro en pacientes alcohólicos podría ser reversible después de varios meses de abstinencia de alcohol, lo cual se encuentra relacionado con la plasticidad neuronal y los cambios que ocurren rápidamente en la arborización dendrítica de las neuronas en el sistema nervioso central $(40,41)$. 
Lo anterior sirve como base para los estudios clínicos que reportan déficit de función específica del lóbulo frontal en alcohólicos crónicos. De acuerdo con diferentes pruebas neuropsicológicas se ha demostrado un leve deterioro cognitivo o de la memoria aproximadamente en un $50-70 \%$ de alcohólicos desintoxicados (42); además de reportarse déficit de atención, memoria de trabajo, velocidad de procesamiento, habilidades visual-espaciales, funciones ejecutivas, impulsividad, aprendizaje, fluidez verbal, todas ellas deterioradas en el alcoholismo $(41,42)$.

A nivel del cerebelo se ha reportado atrofia en la porción anterior del vermis superior y áreas adyacentes de los hemisferios cerebelosos, observándose a nivel macroscópico un adelgazamiento de las laminillas cerebelosas y ensanchamiento de los surcos en el vermis rostral (43); en las zonas afectadas existe pérdida de células de Purkinje y pérdida parchada variable de células granulares, atrofia de la capa molecular y gliosis de Bergmann (44); la incidencia de la degeneración cerebelosa se aproxima a un $27 \%$ de los alcohólicos, presentando un cuadro clínico con inestabilidad troncal, ampliación de la base de sustentación y marcha atáxica (45).

Los cambios cerebrales relacionados con la ingesta de alcohol pueden estar relacionados con la muerte celular, la pérdida de axones, dendritas y sinapsis; de acuerdo con diferentes investigaciones se ha observado un aumento en los valores de difusión media (MD), lo que refleja una mayor tasa de difusión de agua microscópica dentro de los haces de fibras del cuerpo calloso y fórnix; dichos cambios en la MD reflejan alteraciones dentro de las regiones del cerebro que son indicativos de la remodelación de tejidos incluyendo cambios en la microglía, astrocitos, desmielinización, citoarquitectura interrumpida y pérdida axonal dentro de la región afectada (42), lo cual puede estar influenciado por varios factores, incluyendo las barreras tisulares, fluido y espacio extracelular $(46,47)$.

Varias funciones del aparato de Golgi son sensibles a la exposición a EtOH; el cual inhibe el tráfico de las glicoproteínas y provoca un deterioro de la exocitosis y endocitosis (48). Estudios sugieren una relación directa entre la localización del aparato de Golgi y la polarización del crecimiento de las dendritas en la corteza y en el hipocampo (49). El grupo de Lewandowska y col. (2012) estudió en crías de ratas expuestas a $\mathrm{EtOH}$, prenatalmente y durante la lactancia, las alteraciones ultraestructurales en el cerebelo en desarrollo, observando mitocondrias hinchadas, dilatación del retículo endoplasmático rugoso (RER) y de las cisternas del aparato de Golgi (50). En lo que respecta a las mitocondrias de las neuronas corticales expuestas a $\mathrm{EtOH}$, estas presentan una apariencia irregular y desorganización de sus crestas mitocondriales (51). Asimismo, en células bajo influencia de $\mathrm{EtOH}$, se observa gran cantidad de lisosomas y un aumento del lumen del retículo endoplasmático (52). Es sabido que la organización y la dinámica de los microtúbulos (MTs) son críticos para el desarrollo y el funcionamiento correcto de las neuronas, incluyendo el tráfico intracelular y la señalización (53).

\section{Neuroanatomía de la corteza cerebral bajo expo- sición al etanol}

En la intoxicación alcohólica aguda a medida que va aumentando la cifra de alcoholemia, el sujeto intoxicado presenta una fase de hiperexcitabilidad del córtex, para presentar después un síndrome confusional y cerebeloso, hasta llegar a padecer un coma más o menos profundo (2). Esto se debe a que las bajas concentraciones de etanol causan una vasodilatación con aumento del flujo sanguíneo cerebral regional, especialmente en el lóbulo prefrontal, mientras que dosis altas causan una vasoconstricción con una reducción del flujo sanguíneo, asociando de esta manera la euforia que ocurre durante la ingestión aguda de etanol con una activación del córtex prefrontal (54).

En alcohólicos crónicos ha sido descrita una disminución de la densidad neuronal en regiones corticales específicas, demostrando una pérdida neuronal selectiva en la corteza de asociación superior frontal, mientras que ésta no ocurre en la corteza motora, ni en la corteza cingulada anterior; además, diferentes análisis han revelado regiones discretas del cerebro, especialmente regiones de materia blanca en el lóbulo frontal, con una significativa reducción del peso y volumen comparado con los pacientes controles y aún, encontrándose más acentuada en pacientes con encefalopatía de Wernicke (36).

Como se menciona anteriormente las anormalidades del lóbulo frontal se producen en la mayoría de alcohólicos crónicos, lo cual ha sido demostrado según análisis celulares de porciones dorsolaterales de la corteza de asociación frontal, cuya región se cree que está involucrada en el seguimiento y manipulación de la información durante la memoria de trabajo, por lo tanto, la pérdida neuronal en dichas regiones podría explicar muchos déficits del lóbulo frontal comúnmente vistos en 
los alcohólicos, sugiriéndose una baja probabilidad de ser reversibles $(36,55)$.

\section{Cascada de señalización y proteínas sinápticas involucradas en el efecto neurotóxico del etanol}

El EtOH interactúa con ciertas proteínas situadas en la membrana neuronal, responsables de la transmisión de señales y activación de neurotransmisores, lo que lleva a cambios a corto plazo en la regulación de las funciones celulares y a largo plazo en la expresión génica (56).

Canales iónicos, transportadores, receptores, proteínas G y protein-Kinasas son los sitios de acción del etanol con los cuales interactúa y da lugar a cambios en la actividad enzimática, chaperonas y reguladores de la expresión génica (57).

A nivel neuroquímico, el consumo moderado de $\mathrm{EtOH}$ afecta selectivamente la función GABA, glutamatérgica, serotoninérgicas, dopaminérgica, colinérgica y los sistemas neuronales opioides (58). Una de las principales acciones del etanol es su interacción con dos tipos de receptores: $\mathrm{GABA}_{\mathrm{A}}$ (complejo receptor $\mathrm{GABA}_{\mathrm{A}}$-ionóforo $\mathrm{Cl})$ y receptor NMDA del glutamato; estos dos neurotransmisores regulan la excitabilidad de muchas neuronas, GABA tiene un efecto inhibidor del SNC, pertenece a la familia de canales iónicos activados por ligando y consiste en complejos pentaméricos con sitios de unión para agonistas y antagonistas de GABA, benzodiacepinas, barbitúricos y alcoholes, posee un canal de $\mathrm{Cl}^{-}$que permite la hiperpolarización de la membrana neuronal en la mayoría de los casos; por otra parte, el glutamato es uno de los principales excitadores del SNC, especialmente para las respuestas rápidas de las cadenas neuronales $(57,59)$.

Son muchos los estudios que se han documentado a cerca de la activación de receptores $\mathrm{GABA}_{\mathrm{A}}$ por parte del etanol y su antagonismo en la acción del glutamato, por lo cual sus acciones son propiamente las de un depresor del SNC. El etanol potencia las corrientes postsinápticas inhibitorias de GABA en la corteza cerebral, neuronas septales mediales e intermedio laterales y en ciertas neuronas del hipocampo; el mecanismo se basa en un movimiento hiperpolarizante por la entrada de iones $\mathrm{Cl}^{-}$, esto conlleva a una disminución de la excitabilidad de la neurona y de su actividad funcional $(60,61)$.

La acción del etanol sobre los lugares de fijación específcos del receptor $\mathrm{GABA}_{\mathrm{A}}$ es potenciar los compuestos que sobre el actúan, como las benzodiacepinas y barbitúricos, ya que favorece el flujo de cloro al interior de la neurona postsináptica, ocasionando una tolerancia cruzada entre los compuestos (23). El incremento de la activación de los receptores $\mathrm{GABA}_{\mathrm{A}}$ por el etanol, puede ser modulado en parte por la protein kinasa $C$ (62).

En cuanto a la sensibilidad del receptor $\mathrm{GABA}_{\mathrm{A}}$ a las diferentes concentraciones de etanol, la mayoría de los estudios muestran que el alcohol puede potenciar el receptor en concentraciones por encima de $60 \mathrm{mM}$; sin embargo, otros trabajos reportan la existencia de una subpoblación de receptores $\mathrm{GABA}_{\mathrm{A}}$ sensibles a etanol en baja concentración ( $<30 \mathrm{mM}$ ) (63); su ubicación es extrasináptica y media una inhibición tónica lejos del espacio sináptico, en la cual la subunidad $\delta$ se caracteriza por expresarse en su estructura; las subunidades $\delta$ y $\beta_{3}$ parecen ser imprescindibles para que un receptor $\mathrm{GABA}_{\mathrm{A}}$ presente una alta afinidad al etanol, cuando dichas subunidades son intercambiadas por $\mathrm{\gamma}_{2}$ y $\beta_{2}$, respectivamente, la sensibilidad del receptor al alcohol disminuye considerablemente $(23,64)$.

El glutamato es uno de los principales neurotransmisores excitadores cerebrales y juega un papel importante en la iniciación y modulación de procesos neuroadaptativos asociados con el establecimiento de cambios a largo plazo implicados en el aprendizaje y la memoria (65); estos neurotransmisores actúan sobre los receptores NMDA de carácter ionotropo asociados a un canal que permite el flujo de $\mathrm{Na}^{+}, \mathrm{K}^{+}$y Ca${ }^{+2}$, lo que se traduce en una despolarización de la membrana neuronal; la activación del receptor desencadena respuestas derivadas del incremento de calcio intracelular, sin embargo, dada la potencial toxicidad intracelular de las altas concentraciones de $\mathrm{Ca}^{+2}$, este canal se encuentra habitualmente bloqueado por el ion $\mathrm{Mg}^{+2}$, ya que este ion muestra afinidad por un sitio situado en la profundidad del canal impidiendo el flujo intracelular de calcio $(61,65)$.

El etanol mientras actúa de forma aguda sobre este receptor disminuye el flujo de $\mathrm{Ca}^{+2}$ a través del canal en un número de áreas del cerebro, cuyos efectos parecen estar restringidos a zonas circunscritas del SNC, incluyendo el neocórtex, el locus coeruleus, posiblemente involucrados en la amnesia y las reacciones inducidas por la abstinencia al etanol, respectivamente (65). La activación del receptor NMDA es responsable de los fenómenos de potenciación a largo plazo (long-term potentiation, LPT) involucrados en los procesos de aprendizaje y memoria, los cuales pueden ser inhibidos por los efectos del etanol (33). 
Al igual que el receptor $\mathrm{GABA}_{\mathrm{A}}$, existe una variabilidad en la sensibilidad del etanol sobre el receptor NMDA que depende de la composición de las subunidades de dicho receptor, de esta manera las combinaciones NMDAR1/NMDAR2 (NR1/NR2A) y especialmente NR1/ NR2B son más sensibles a la inhibición por etanol (65).

\section{Vías inmunológicas relacionadas con la neuro- toxicidad del etanol}

Se ha demostrado que los genes y las vías que participan en las respuestas al estrés se encuentran aumentados en los alcohólicos; el consumo crónico y exceso de EtOH conduce a efectos dramáticos en estos pacientes, ya que se ha observado una disminución en el tamaño del hipocampo, región clave relacionada con el aprendizaje; estos efectos se atribuyen a una combinación de neurodegeneración y decremento en la neurogénesis, lo cual está relacionado con la inflamación inducida por el etanol a través de receptores Toll-like y la activación de la vía NF-kB, (factor nuclear potenciador de las cadenas ligeras kappa de las células $B$ activadas) que juegan un papel en la regulación de la inflamación, la respuesta inmune y la apoptosis (66-68).

El EtOH activa la inflamación a través de la vía TLR4 y NF-kB; los receptores Toll-like 4 de la neuroglia desencadenan la producción de mediadores inflamatorios, lo cual está asociado con la desmielinización y daño neuronal; análisis inmunohistoquímicos revelan una alteración en la morfología de la mielina por una disminución en el número de fibras de la Proteína Básica de Mielina (MBP), ocasionando la muerte de oligodendrocitos, además de promover la disminución de la Proteína Proteolipídica (PLP), uno de los componentes principales de la mielina y por tanto importante en la transmisión de impulsos (68-70) (Figura 2 y 3).

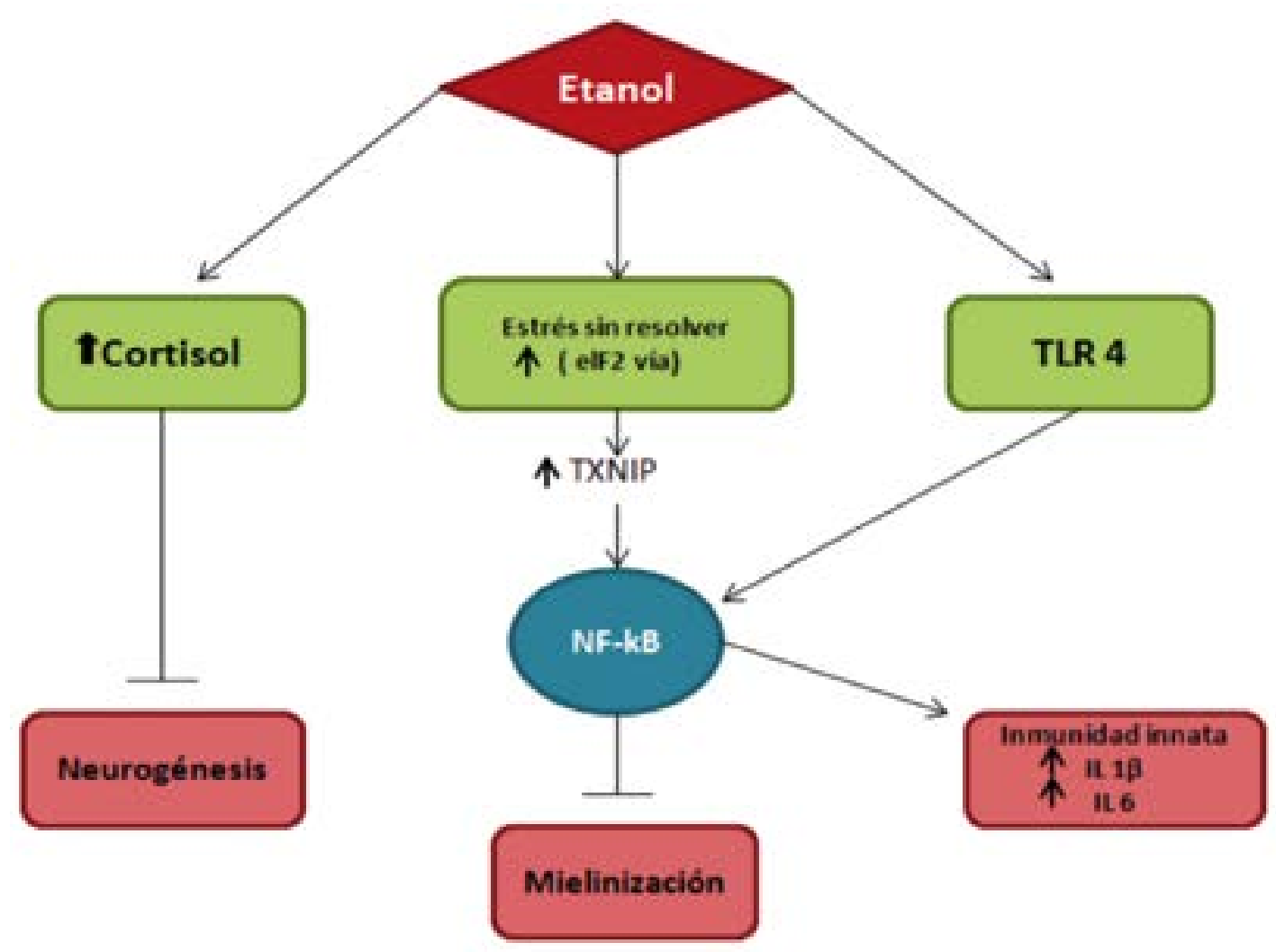

Figura 2. Principales vías afectadas por el etanol. La ingesta de etanol activa la vía NF-kB a través del receptor Toll-like 4 (TLR4), lo que a su vez aumenta la actividad de la respuesta inmune innata e inhibe la neurogénesis hipocampal y la activación de otros genes (Modificado de: McClintick J. et al. 2013). 


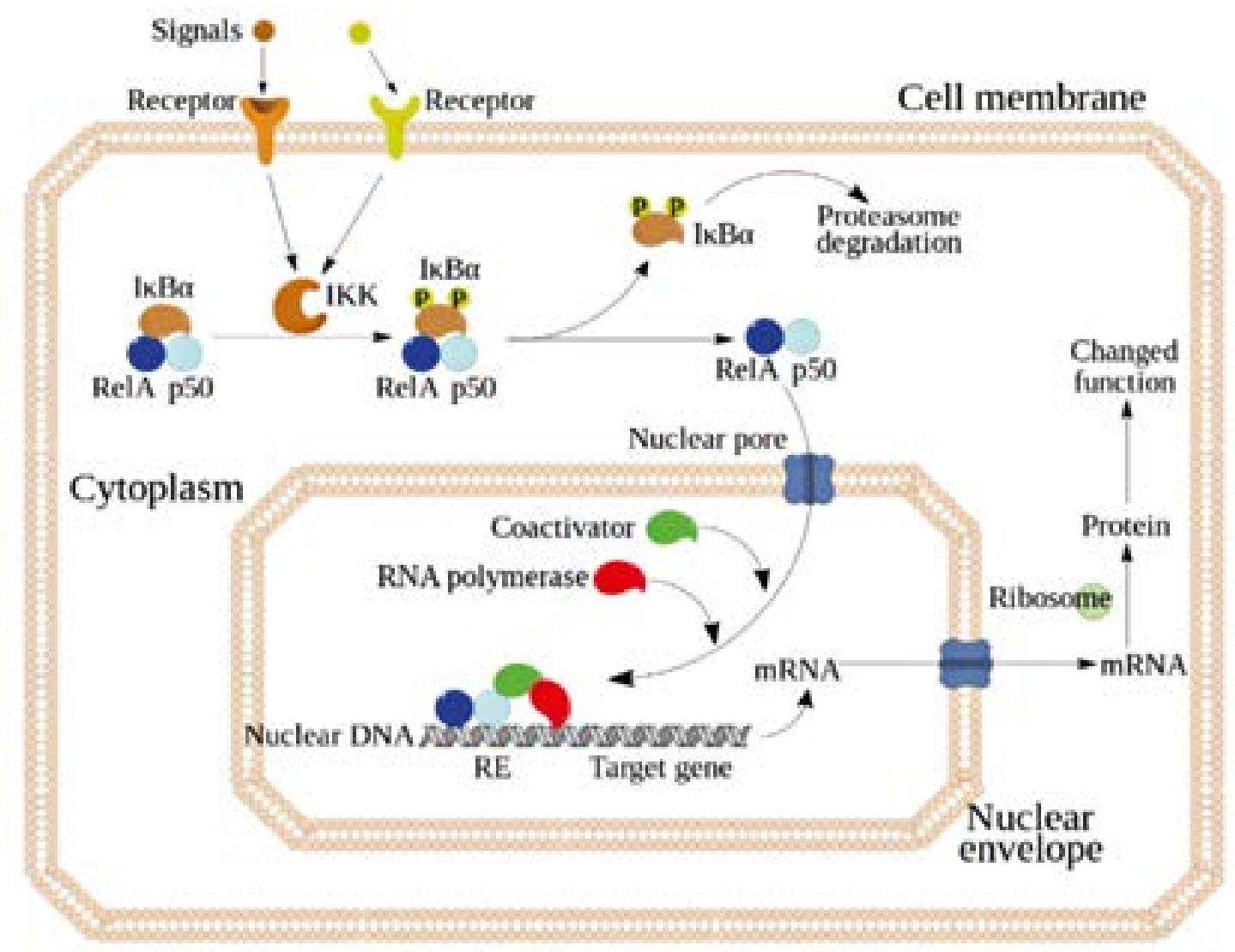

Figura 3. Mecanismo de acción del NF-KB. En la imagen, se muestra como ejemplo el heterodímero NF$\mathrm{KB}$ entre el Rel y las proteínas $\mathrm{p50}$. En su estado inactivo, el NF-KB se encuentra en el citosol formando un complejo con la proteína inhibitoria IKBa. A través de receptores integrales de membrana, una variedad de señales extracelulares activa la enzima quinasa ikB (IKK). La IKK, a su vez, fosforila la proteína IKBa, que da lugar a la ubiquitinación, disociación del complejo IKBa -NF-KB, y la degradación eventual de IKBa por el proteosoma. Posteriormente la NF-KB activada entra en el núcleo donde se une a secuencias específicas llamadas elementos de respuesta (RE). El complejo ADN/NF- $\mathrm{K} B$ da lugar a otras proteínas como los coactivadores $A R N$ polimerasa, que transcribe $A D N$ en $A R N$ mensajero, que a su vez se transforma en proteínas que provocan un cambio en la función de la célula (71-72).

\section{Apoptosis}

Por otro lado, se ha manifestado que muchos órganos humanos son capaces de metabolizar el etanol a través de un mecanismo no oxidativo formando etilésteres de ácidos grasos, mediante la acción catalizada por la enzima etil ester sintetasa (73); cuyos efectos fisiológicos consisten en la afectación de la capacidad oxidativa de la mitocondria, además de su capacidad para desordenar las membranas celulares (74). Dichos metabolitos podrían explicar la lesión tisular observada en órganos que carecen de metabolismo oxidativo, además de daño cerebral originado por consumo crónico de cantidades abundantes de $\mathrm{EtOH}(29,74)$.
Observaciones realizadas a nivel de la corteza cerebral han reportado que el consumo crónico de alcohol promueve la expresión de proteínas proapoptóticas como Bax y Omi/HtrA2 produciendo una caída del potencial transmembranal con el consecuente aumento en la permeabilidad de la misma; por otro lado, el consumo de etanol genera una represión de expresión de proteínas anti-apoptóticas: Bcl-2 y ARC (75).

La activación de moléculas señales de estrés p38 y JNK que pertenecen a la familia de proteínas serina/treonina (MAPK) implicadas en una amplia gama de transducción de señales, disminuyen la fosforilación de Akt y GSK3 , lo que demuestra el papel del acetaldehído inducido 
por la ingesta de alcohol en el daño cerebral (76). La cascada MAPK p38 es principalmente funcional cuando las células responden a diversos estímulos de estrés, así como ligandos que activan receptores relacionados con la apoptosis; de igual forma la cascada JNK desempeña un papel importante en la respuesta al estrés celular mediante inducción de la apoptosis (77-79).

\section{Conclusiones}

El EtOH es sin duda la droga más extendida y tolerada a nivel social. Sus efectos sobre el sistema nervioso pueden ser consecuencia de un consumo agudo y excesivo o secundario a un consumo crónico, en relación a un estado de adicción y dependencia al mismo. La toxicidad sobre el sistema nervioso es consecuencia directa de la agresión del etanol o por diferentes trastornos metabólicos ligados a la toxicidad del mismo sobre otros órganos.

A nivel del sistema nervioso central se observan alterados sistemas de neurotransmisión y factores de crecimiento nervioso. Los hallazgos neuropatológicos en el sistema nervioso central ponen de manifiesto lesiones corticales y subcorticales (tipo Wernicke-Korsakokoff, mielinosis). La aparición de sintomas y signos de disfunción frontal indican lesión preferencial del córtex a este nivel.

a aparición de uno u otro cuadro neurológico y el pronóstico del mismo dependerán de la dosis de etanol consumida, del tiempo de exposición, la existencia de otras patologías previas y el correcto manejo terapéutico, entre otros factores; en la práctica clínica coexiste el daño a distintos niveles.

La neurotoxicidad del alcohol se debe a la activación del sistema inmunitario innato en el cerebro, que promueve compuestos inflamatorios que serían los responsables del daño neural. El alcohol activa los receptores denominados "toll-like" o "TLRs", proteínas ancestrales responsables de la respuesta inmune innata y de la defensa. Estos receptores se encuentran en las células del sistema inmune, reconocen a un gran número de agentes patógenos y su interacción provoca una rápida respuesta y la producción de compuestos tóxicos e inflamatorios, su sobreactivación puede causar inflamación crónica y lesiva.

Las células gliales juegan un papel crucial en la respuesta inmune, liberando citoquinas y actuando de células mediadoras en la inflamación. Su activación por estímulos tóxicos puede causar respuestas anómalas y contribuir de esta forma a la neurodegeneración y al daño cerebral.
Los receptores TLR4 activan factores proinflamatorios como reacción ante el daño neural. La neurotoxicidad del etanol está, en parte, mediada por mecanismos de neuroinflamación, igual que ocurre en ciertas enfermedades neurodegenerativas.

Se ha demostrado que la ingesta de altas dosis de alcohol de forma intermitente durante la adolescencia, como sucede en el patrón de consumo de los jóvenes que beben principalmente los fines de semana, está asociada a la muerte neuronal y a la aparición de dificultades en los procesos cognitivos y de aprendizaje de los adolescentes.

\section{Conflicto de Intereses}

Los autores de este artículo declaran no tener ningún tipo de conflicto e intereses.

\section{Fuente de Financiación}

Ninguna

\section{Referencias}

1. Velasco A. Farmacología y toxicología del alcohol etílico o etanol. An Real Acad Med Cir Vall. 2014; 51:242248. ISSN 0210-6523

2. Estruch R. Efectos del alcohol en la fisiología humana. Adicciones. 2002;14(1):43-61. DOI: http://dx.doi. org/10.20882/adicciones.519

3. Secretaría de Salud del Estado de Veracruz. Guía de diagnóstico y tratamiento de intoxicación por alcohol etílico o etanol. Centro de información toxicológica de Veracruz. http://web.ssaver.gob.mx/citver/files/2014/11/ Intoxicación-por-Alcohol-Etílico-o-Etanol.pdf

4. World Health Organization. Glosario de términos de alcohol y drogas. Madrid: Ministerio de Sanidad y Consumo, Centro de Publicaciones; 1994. http://www. who.int/substance_abuse/terminology/lexicon_alcohol_ drugs_spanish.pdf

5. Babor TF, Higgins-Biddle JC, Saunders JB, Monteiro MG. AUDI: Cuestionario de Identificación de los Trastornos debidos al Consumo de Alcohol. Ginebra: Organización Mundial de la Salud, Departamento de Salud Mental y Dependencia de Sustancias; 2001. http:// www.who.int/substance_abuse/activities/en/AUDITmanualSpanish.pdf

6. Anderson P., Gual A., Colon J. Alcohol y atención primaria de la salud: informaciones clínicas básicas para la identificación y el manejo de riesgos y problemas. Organización Panamericana de la Salud. 2008. ISBN 9789275328569. http://www.who.int/substance abuse/publications/alcohol_atencion_primaria.pdf

7. Téllez J, Cote M. Alcohol Etílico: Un tóxico de alto riesgo para la salud humana socialmente. Rev Fac 
Med. 2006;54(1):32-47.ISSN En línea: 2357-3848

8. Repetto M. Toxicología del alcohol etílico. En: Toxicología avanzada, Capítulo 11. Ediciones Díaz de Santos, S.A. 1995. Pp.425. ISBN 9788479782016

9. Guadagnoli, T. ¿La neurotoxicidad del etanol depende del estadío de diferenciación neuronal? Estudio en cultivos primarios de neuronas corticales. Tesis Doctoral. Biblioteca Digital de la Facultad de Ciencias Exactas y Naturales. Universidad de Buenos Aires. 2014.

10. Rehm J, Monteiro M. Alcohol consumption and burden of disease in the Americas-implications for alcohol policy. Rev Panam Salud Pública. 2005;18(4-5):241-8. http:// dx.doi.org/10.1590/S1020-49892005000900003

11. Téllez J. Aspectos toxicológicos, psicológicos y sociales. Relacionados con el consumo de bebidas alcohólicas. Capítulo: Propiedades fisicoquímicas, toxicocinética y mecanismos de toxicidad. Editorial: U. Nacional de Colombia. 2012. ISBN: 9789587617870

12. Redolar D. Cerebro y adicción. Editorial UOC. 2008. Pp.513. ISBN 9788497887472

13. Carrillo R, Aguirre D, Villanueva L, Lelo E, García L. Intoxicación por etilenglicol. Reporte de un caso y revisión de la literatura. Acta Médica Grupo Ángeles. 2006; 4(4):243-248.

14. Loría J, Bermeo L, Ramírez A, Fernández D. Intoxicación por metanol, reporte de un caso. Archivos de medicina de Urgencia de México. 2009;1(2):67-73.

15. Aragón C., Miquel M., Correa M., Segura S. Alcohol y metabolismo humano. Adicciones. 2002;14(1):2342. ISSN 0214-4840

16. Díez P., Gallego M., Fernández J., Ganzo M. Intoxicaciones agudas por alcohol, otras drogas y fármacos psicoactivos. Medicine. 2015;11(89):5314-23. ISSN 0304-5412

17. Riveros-Rosas H, Julian-Sanchez A, Pinã E.. Enzymology of ethanol and acetaldehyde metabolism in mammals. Arch Med Res Winter. 1997;28(4):453-71. PMID: 9428569

18. Lieber CS.. Ethanol metabolism, cirrhosis and alcoholism. Clinica Chimica Acta 257, 1997;59-84. PMID: 9028626

19. Álvarez-González FJ, Del Río Gracia C. "Farmacología del alcohol". En: Manual SET de alcoholismo $-1^{\text {a }}$ Ed. - Madrid: Médica Panamericana. 2003. Pp.109-129. ISBN: 84-7903-873-X

20. Thomasson HR, Crabb DW, Edenberg HJ, Li TK, Hwu HG, Chen CC, et al. Low frequency of the ADH2*2 allele among Atayal natives of Taiwan with alcohol use disorders. Alcohol Clin Exp Res. 1994;18(3):640-3. http://doi:10.1111/j.1530-0277.1994.tb00923.x

21. Tanaka F, Shiratori Y, Yokosuka O, Imazeki F, Tsukada Y, Omata M. High incidence of ADH2*1/ALDH2*1 genes among Japanese alcohol dependents and patients with alcoholic liver disease. Hepatology. 1996;23:234239. DOI: 10.1053/jhep.1996.v23.pm0008591846

22. Flórez J., Armijo J., Mediavilla A. Farmacología humana. Cuarta edición. Editorial Masson, S.A. 2005.
Pp.28. eBook ISBN: 9788445825235

23. Arias R. Reacciones fisiológicas y neuroquímicas del alcoholismo. Diversitas. 2005:2(2);138-147. ISSN Online: 2256-3067

24. Romero C, Galindo F, Galicia S, Flores A. GABA: ¿Dualidad funcional? Transición durante el neurodesarrollo. Rev Neurol. 2011:52(11);665-675. PMID: 21563118

25. Rengifo A., Tapiero C., Spinel C. Receptores GABAA (ácido $\mathrm{Y}$-aminobutírico) y su relación con la dependencia al alcohol. Ingeniería y Ciencia. 2005:1(1);77-96. EISSN: 2256-4314

26. Oviedo H., Arboleda P. Fisiopatología y tratamiento del síndrome de abstinencia de alcohol. Universitas Médica. 2006;47(2):112-120. ISSN: 0041-9095

27. Koolman J., Klaus H. Bioquímica: texto y atlas. $3^{\text {a }}$ edición. Madrid: Médica Panamericana. 2004. Pp.320. ISBN: 84-7903-724-5

28. Muller W. Bioquímica: Fundamentos para Medicina y ciencias de la vida. Editorial Reverté. 2008. Pp.624. ISBN: 978-84-291-7393-2

29. Rodés J., Benhamou J., Bircher J., Mclntyre N., Rizzetto M. Tratado de hepatología clínica. Segunda Edición. Tomo II. Editorial Masson. 2001. Pp.1313. ISBN 84458-0966-0

30. Bataller R. Toxicología clínica. Imprenta Romeu. Universitat de Valencia. 2004. Pp.56. ISBN: 9788437060156

31. Martínez A., Rábano A. Efectos del Alcohol etílico sobre el Sistema Nervioso. Rev Esp Patol. 2002;35(1):63-76

32. Souza M., Macharro S. Alcoholismo, conceptos básicos. México: Manual Moderno.1988. ISSN Online: 22563067

33. Koechling UM, Amit Z. Relationship between blood catalase activity and drinking history in a human. Alcohol. 1992;27(2):181-8. PMID: 1524610

34. Givens B., McMahon K. Ethanol suppresses the induction of long-term potentiation in vivo. Brain Research. 1995;688:27-33. PMID: 8542319

35. Raskin N., Sokoloff L. Enzymes catalysing ethanol metabolism in neural and somatic tissues of the rat. J. Neurochem. 1972;19(2):273-282. DOI: 10.1111/j.14714159.1972.tb01337.x

36. Gill K., Menez F., Lucas D., Deitrich A. Enzymatic production of acetaldehyde from ethanol in rat brain tissue. Alcohol Clin Exp Res. 1992;16:910-15. PMID: 1443429

37. Kril J., Halliday G., Svoboda M., Cartwright H. The cerebral cortex is damaged in chronic alcoholics. Neuroscience. 1997;79(4):983-998. PMID: 9219961

38. Walker D., Hunter B., Abraham W. Neuroanatomical and functional deficits subsequent to chronic ethanol administration in animals. Alcohol Clin Exp Res. 1981;5(2):267-82. PMID: 7018310

39. Harper C., Kril J., Daly J. Are we drinking our neurons away?. British Medical Journal. 1987;294:534-536. PMCID: PMC1245575

40. Gopal D., Robert B. Neura Transplantation and Regeneration. Springer-Verlag New York Inc. 1986. pp. 75. 
41. Stavro K, Pelletier J, Potvin S. Widespread and sustained cognitive deficits in alcoholism: a meta-analysis. Addiction Biology. 2013;18:203-13. DOI: 10.1111/j.13691600.2011.00418.x

42. Berman M, Marinkovic'K. Alcohol: effects on neurobehavioral functions and the brain. Neuropsychology Review. 2007;17:239-57. DOI: 10.1007/s11065-007-9038-6

43. Trivedi R., Bagga D., Bhattacharya D., Kaur P., Kumar P., Khushu S., et al. White matter damage is associated with memory decline in chronic alcoholics: A quantitative diffusion tensor tractography study. Behavioural Brain research. 2013;250:192-198. DOI: 10.1016/j. bbr.2013.05.001

44. Victor M, Adams RD, Mancall EL. A restricted form of cerebellar cortical degeneration occurring in alcoholic patients. Arch Neurol 1959;71:579-688. doi:10.1001/ archneur.1959.03840060001001

45. Stephen C., Harper C., Kril J. A Quantitative Histological Study Of The Cerebellar Vermis In Alcoholic Patients. Brain. 1987;110:301-14. https://doi.org/10.1093/ brain/110.2.301

46. Martínez M., Bermejo E., Rodríguez E. Evolución temporal y por comunidades autónomas del consumo de diferentes cantidades de alcohol durante el embarazo. Med Clin (Barc). 2003;120(14):535-41. DOI: 10.1016/ S0025-7753(03)73766-X

47. Beaulieu C. The basis of anisotropic water diffusion in the nervous system-a technical review. NMR Biomed. 2002; 15:435-455. PMID: 12489094

48. Pfefferbaum A., Sullivan E. Disruption of Brain White Matter Microstructure by Excessive Intracellular and Extracellular Fluid in Alcoholism: Evidence from Diffusion Tensor Imaging. Neuropsychopharmacology. 2005;30:423-432 PMID: 15562292

49. Marín MP, Esteban-Pretel G, Ponsoda X, Romero AM, Ballestín R, López C, et al. Endocytosis in cultured neurons is altered by chronic alcohol exposure. Toxicol Sci. 2010;115(1):202-13. PMID: 20133374

50. Matsuki T, Matthews RT, Cooper JA, van der Brug MP, Cookson MR, Hardy JA, et al. Reelin and stk25 have opposing roles in neuronal polarization and dendritic Golgi deployment. Cell. 2010;143:826-836. PMCID: PMC3033572

51. Lewandowska E, Stępień T, Wierzba-Bobrowicz T, Felczak P, Szpak GM, Pasennik E. Alcohol-induced changes in the developing cerebellum. Ultrastructural and quantitative analysis of neurons in the cerebellar cortex. Folia Neuropathol. 2012;50(4):397-406. DOI: https:// doi.org/10.5114/fn.2012.32374

52. Tavares MA, Paula-Barbosa MM. Mitochondrial changes in rat Purkinje cells after prolonged alcohol consumption a morphologic assessment. J Submicorsc Cytol. 1983; 15:713-720. PMID: 6683761

53. Dlugos CA. Ethanol-related increases in degenerating bodies in the Purkinje neuron dendrites of aging rats. Brain Res. 2008 24;1221:98-107. doi: 10.1016/j. brainres.2008.05.015

54. Hoogenraad CC, Bradke F. Control of neuronal polarity and plasticity - a renaissance for microtubules. Trends Cell Biol. 2009;19:669-676. PMID: 19801190

55. Tiihonen J., Kuikka J., Hakola P., Paanila J., Airaksinen J., Eronen M., Hallikainen T. Acute etanol-induced changes in cerebral blood flow. Am J Psychiatri. 1994;151(10):1505-8. PMID: 8092344

56. Petrides M. Lateral frontal cortical contribution to memory. Seminars in The Neurosciences. 1996;8:57-63. http://dx.doi.org/10.1006/smns.1996.0008

57. Diamond I., Gordon A. Cellular and molecular neuroscience of alcoholism. Physiological Reviews. 1997;77(1):120. PMID: 9016298

58. Faingold L., N`Gouemo P., Riaz A. Ethanol and neurotransmitter interactions: from molecular to integrative effects. Prog Neurobiol 1998;55:509-535. PMID: 9670216

59. Eckardt M., File S., Luigi G., Grant K., Guerri C., Hoffman P., et al., Tabakoff B. Effects of moderate alcohol consumption on the central nervous system. Alcoholism: Clinical and Experimental Research. 1998;22(5):9981040. PMID: 9726269

60. Bustamante E. El sistema nervioso. Desde las neuronas hasta el cerebro humano. Editorial Universidad de Antioquia. Medellín. 2004. Pp.113. ISBN: 978-958-714-073-6.

61. Signore A., Yeh H. Chronic Exposure to Ethanol Alters GABAA Receptor-Mediated Responses of Layer II Pyramidal Cells in Adult Rat Piriform Cortex. Journal of Neurophysiology. 2000;84(1):247-254.

62. Ayesta F. Bases bioquímicas y neurobiológicas de la adicción al alcohol. Adicciones. 2002;14(1):63-78. DOI: http://dx.doi.org/10.20882/adicciones.520

63. Weiner J., Zhang L., Carlen P. Potentiation of GABAA-mediated synaptic current by ethanol in hippocampal CA1 neurons: possible role of protein kinase C. J Pharmacol Exp Ther. 1994;268(3):1388-95.PMID: 8138953

64. Wei W., Coutinho L., Mody I. Low Ethanol Concentrations Selectively Augment the Tonic Inhibition Mediated by $\delta$ Subunit-Containing GABAA Receptors in Hippocampal Neurons. The Journal of Neuroscience. 2004;24(38):8379-8382. DOI: https://doi. org/10.1523/JNEUROSCI.2040-04.2004

65. Wallner M., Hanchar H., Olsen R. Ethanol enhances $\alpha 4 \beta 3 \delta$ and $\alpha 6 \beta 3 \delta \mathrm{Y}$-aminobutyric acid type A receptors at low concentrations known to affect humans. PNAS. 2003;100(25):15218-15223. doi: 10.1073/ pnas. 2435171100

66. Wirkner K., Poelchen W., Koles L., Muhlberg K., Scheibler P., Allgaier C., Illes P. Ethanol-induced inhibition of NMDA receptor channels. Neurochemestry International. 1999;35:153-162. PMID: 10405999

67. Agartz I., Momenan R., Rawlings R., Kerich M., Hommer D. Hippocampal volume in patients with alcohol dependence. Archives of General Psychiatry. 1999;56:356363. PMID: 10197833 
68. Monje M., Toda H., Palmer T. Inflammatory blockade restores adult hippocampal neurogenesis. Science. 2003;302:1760-1765. PMID: 14615545

69. Alfonso S., Pascual M., Gomez U., Pascual M., Renau J., Guerri C. Toll-like receptor 4 participates in the myelin disruptions associated with chronic alcohol abuse. Glia. 2012;60:948-964. DOI: 10.1002/glia.22327

70. McClintick J., Xuei X., Tischfield J., Goate A., Foroud T., Wetherill L., et al., Edenberg H. Stress-response pathways are altered in the hippocampus of chronic alcoholics. Alcohol. 2013;47:505-515.PMCID: PMC3836826

71. Morris S., Eaves D., Smith A., Nixon K. Alcohol inhibition of neurogenesis: A mechanism of hippocampal neurodegeneration in an adolescent alcohol abuse model. Hippocampus. 2010;20(5):596-607. PMCID: PMC2861155

72. Gilmore TD. Introduction to NF-KB: players, pathways perspectives. Oncogene. 2006;25 (51):6680-4. DOI: 10.1038/sj.onc. 1209954

73. Brasier AR. The NF-kB regulatory network". Cardiovasc. Toxicol. 2006;6(2):111-30. PMID: 17303919

74. Mogelson S, Lange LG. Nonoxidative ethanol metabolism in rabbit myocardium purification to homogeneity of fatty acid ethyl ester synthase. Biochemistry 1984;23:407581. PMID: 6487591

75. Elvir J. Radicales libres, alcoholismo y daño hepático. Revista médica Hondureña. 1994;62:76-82.

76. Ren J., Babcock S., Li Q., Huff A., Li S., Doser T. Aldehyde dehydrogenase-2 transgene ameliorates chronic alcohol ingestion-induced apoptosis in cerebral cortex. Toxicology Letters. 2009;187:149-156. PMCID: PMC2680775

77. Rodríguez M. Papel De P38-Mapk En El Proceso De Apoptosis Inducido Por Cisplatino En Células Epiteliales De Túbulo Renal. Tesis Doctoral. Universidad Complutense de Madrid. 2008.

78. Miñana R., Climent E., Barettino D., Segui J., Renau-Piqueras J., Guerri C. Alcohol exposure alters the expression pattern of neural cell adhesion molecules during brain development.J Neurochem. 2000;75(3):954-64. PMID: 10936176

79. Perkins ND. Integrating cell-signalling pathways with NF-KB and IKK function. Nat. Rev. Mol. Cell Biol. 2007;8(1):49-62. DOI: $10.1038 / \mathrm{nrm} 2083$ 